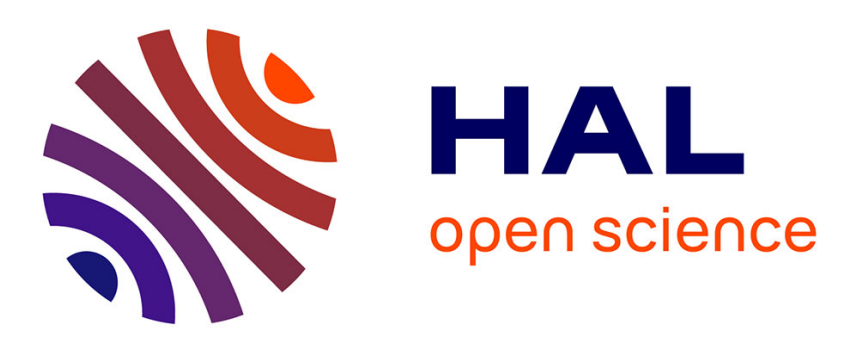

\title{
Pour une cartographie des parties prenantes fondée sur leur engagement: une application aux sociétaires d'une banque mutualiste française
}

Carine Girard, André Sobczak

\section{- To cite this version:}

Carine Girard, André Sobczak. Pour une cartographie des parties prenantes fondée sur leur engagement: une application aux sociétaires d'une banque mutualiste française. Revue management \& avenir, 2010, (33), pp.157-174. 10.3917/mav.033.0157 . hal-00771148

\author{
HAL Id: hal-00771148 \\ https://hal.science/hal-00771148
}

Submitted on 11 Apr 2013

HAL is a multi-disciplinary open access archive for the deposit and dissemination of scientific research documents, whether they are published or not. The documents may come from teaching and research institutions in France or abroad, or from public or private research centers.
L'archive ouverte pluridisciplinaire HAL, est destinée au dépôt et à la diffusion de documents scientifiques de niveau recherche, publiés ou non, émanant des établissements d'enseignement et de recherche français ou étrangers, des laboratoires publics ou privés. 


\title{
Pour une cartographie des parties prenantes fondée sur leur engagement : une application aux sociétaires d'une banque mutualiste française
}

\author{
par Carine Girard ${ }^{54}$ et André Sobczak ${ }^{55}$
}

\section{Résumé}

L'objectif de cet article est de proposer une nouvelle classification des parties prenantes fondée sur le concept d'engagement. L'engagement est analysé comme un processus d'apprentissage organisationnel et social entre les dirigeants et leurs parties prenantes. Cet engagement est une condition nécessaire - même si elle n'est pas suffisante - à la responsabilité globale de l'entreprise. Appliqué au sociétariat d'une banque mutualiste française pour lui permettre d'identifier leurs sociétaires plus ou moins engagés, cette nouvelle cartographie peut aider ses dirigeants, d'une part à adapter leurs relations au degré d'engagement de chaque groupe de sociétaires identifiés, et d'autre part à adapter leurs produits financiers et leur communication pour favoriser cet engagement auprès du plus grand nombre de ces groupes.

\section{Abstract}

The aim of this article is to propose a new classification of stakeholders based on the concept of commitment. Commitment is analysed as a social and organisational learning process shared between managers and their stakeholders. This commitment is a necessary condition - even if it is not sufficient - to the corporate social responsibility of the business. Applied to the membership of a French mutual bank in order to identify the members' varying levels of commitment, this new mapping technique can help managers to adapt their practices to the degree of commitment of each identified group of members, and to modify their financial products and communications to foster commitment among as many of these groups as possible.

Un nombre croissant d'entreprises de tous les secteurs et de toutes les tailles cherchent à améliorer l'impact de leurs activités sur l'environnement économique, social et naturel. Beaucoup mettent en œuvre des projets, parfois ambitieux, pour contribuer au développement économique de leur territoire, à la réduction des injustices sociales ou à la protection de l'environnement. Certaines ont commencé à inscrire ces projets dans une véritable stratégie de responsabilité globale définie comme un processus d'apprentissage engagé par l'entreprise

54. Carine Girard, Audencia Nantes School of Management, Institut pour la Responsabilité Globale dans l'Entreprise, cgirard@ audencia.com

55. André Sobczak, Audencia Nantes School of Management, Institut pour la Responsabilité Globale dans l’Entreprise, asobczak@ audencia.com 


\section{management} \& avenir

et ses parties prenantes pour contribuer au développement de la performance économique, sociale et environnementale aussi bien de l'entreprise que de ses parties prenantes (Berthoin Antal et Sobczak, 2004). La légitimité et l'efficacité de ces démarches, comme celles de tout projet dans le domaine de la responsabilité globale, repose donc de manière importante sur la qualité des relations que l'entreprise réussit à nouer avec ses parties prenantes et en particulier sur sa capacité à créer des rapports de confiance permettant de définir des objectifs communs et de favoriser les changements nécessaires à l'atteinte de ceux-ci. Ce qui compte ce n'est pas seulement le contenu des pratiques mises en œuvre, mais également la manière dont elles sont conçues et réalisées, les démarches partagées avec les parties prenantes étant préférées de plus en plus aux démarches unilatérales des managers des entreprises.

Dans cette perspective, les premières étapes d'une démarche de responsabilité globale pour les managers consistent à identifier les différentes parties prenantes de leur entreprise et leurs représentants, puis à analyser leurs intérêts et attentes à l'égard de l'entreprise. Au cours des trente dernières années, de nombreuses recherches en gestion ont par conséquent été menées sur la manière d'analyser et cartographier les parties prenantes et leurs intérêts ainsi que sur la manière dont les managers de l'entreprise peuvent gérer les relations avec celles-ci (Mercier, 2006). Tout en s'appuyant sur ces recherches, l'objectif de cet article est de les faire évoluer, en montrant l'intérêt d'établir une cartographie des parties prenantes en fonction de leur engagement à l'égard de l'entreprise et à l'égard des principes et valeurs de la responsabilité globale. Classer les différentes parties prenantes selon leur degré d'engagement présente notamment l'avantage de souligner la nécessité pour l'entreprise de réactualiser à intervalles réguliers la cartographie des parties prenantes et de l'analyser en tenant compte de la dynamique de ses relations avec les parties prenantes. Après avoir présenté les principes de cette nouvelle cartographie, il s'agit de mettre en évidence son intérêt à partir d'un premier cas d'entreprise dans lequel elle a été testée.

Dans une première partie, nous allons donc présenter notre nouveau cadre d'analyse visant à établir une cartographie dynamique des parties prenantes d'une entreprise fondée sur leur engagement organisationnel et sociétal. Après une revue de la littérature sur l'analyse et le management des parties prenantes, cette première partie montre les limites des principales cartographies actuellement mobilisées dans ce domaine et propose ensuite une cartographie renouvelée et plus dynamique. Dans une deuxième partie, nous allons analyser les apports de cette nouvelle cartographie et son opérationnalité, en nous appuyant sur une étude visant à analyser les intérêts et attentes des sociétaires d'une banque mutualiste française à l'égard de la responsabilité globale. Nous présenterons la méthodologie de cette étude et ses principaux résultats avant de conclure sur les enseignements à tirer de ce premier test de la cartographie dynamique des parties prenantes fondée sur l'engagement. 


\section{Pour une cartographie des parties prenantes fondée sur leur engagement: une application aux sociétaires d'une banque mutualiste française}

Le choix de cette première étude de cas est doublement intéressant. D'une part, les sociétaires des banques mutualistes sont difficiles à ranger dans les catégories des cartographies de parties prenantes les plus répandues, dans la mesure où ils sont à la fois des actionnaires et des clients, voire parfois des salariés de la banque, confirmant donc l'utilité d'une nouvelle cartographie qui dépassent ces statuts. D'autre part, encore peu de recherches en management s'intéressent pour l'instant au secteur mutualiste et coopératif, même dans le domaine de la responsabilité globale, alors que la gouvernance particulière de ce secteur présente un modèle qui peut sembler favorable à une meilleure intégration des enjeux économiques, sociaux et environnementaux que dans les entreprises du secteur capitalistique.

\section{Pour une nouvelle cartographie des parties prenantes}

A partir d'une revue de la littérature sur l'analyse et le management des parties prenantes, nous cherchons à montrer dans cette première partie les limites des cartographies actuellement mobilisées dans ce domaine pour proposer une nouvelle cartographie dynamique des parties prenantes fondée sur leur engagement à l'égard de l'entreprise et à l'égard des principes et valeurs de la responsabilité globale.

\section{1.Des parties prenantes aux parties engagées}

Selon la définition la plus communément diffusée, les parties prenantes d'une organisation sont l'ensemble des individus et groupes qui affectent ou qui sont affectés par la réalisation de ses objectifs (Freeman, 1984). Certains auteurs soulignent que ces individus ou groupes contribuent ainsi, volontairement ou non, à la capacité de l'organisation à créer de la valeur et à ses activités et qui donc en bénéficient et/ou en subissent les risques (Post, Preston \& Sachs, 2002). En général, ces définitions sont complétées par une liste non exhaustive des parties prenantes d'une entreprise qui comprend le plus souvent les salariés, les actionnaires, les clients, les fournisseurs, les pouvoirs publics et les ONG. Pour construire une démarche de responsabilité globale, les dirigeants doivent commencer par identifier les différentes parties prenantes de l'entreprise (Freeman et Gilbert, 1987), leurs attentes respectives à l'égard de cette entreprise ainsi que leurs stratégies (Frooman, 1999). Le succès d'une démarche de responsabilité globale repose ensuite sur une analyse approfondie des intérêts et des pouvoirs de ceux-ci (Wood, 1994). Les recherches menées dans ce domaine soulignent que les différents membres de chaque type de parties prenantes ne constituent pas une catégorie homogène (Wolfe et Puttler, 2002) et ne poursuivent pas nécessairement les mêmes objectifs à l'égard de l'entreprise (Wood, 1994 ; Scholes et Clutterbuck, 1998), rendant ainsi essentielle une classification des membres des différentes catégories de parties prenantes (Freeman et Gilbert, 1987). 


\section{3 \\ management \& avenir}

La classification la plus connue est celle qui distingue d'un côté les parties prenantes primaires ou contractuelles, correspondant aux actionnaires, aux salariés, aux clients et aux fournisseurs et, de l'autre côté les parties prenantes secondaires, comme notamment les ONG, les pouvoirs publics, les médias ou les concurrents (Caroll, 1989 ; Donaldson et Preston, 1995). D'autres auteurs (Mitchell, Agle et Wood, 1997) proposent une classification des parties prenantes selon leur pouvoir d'influence, leur degré de légitimité et le degré d'urgence des intérêts qu'elles défendent face à une situation d'exposition à un risque. Ces typologies qui se complètent plus qu'elles ne s'opposent ont en commun de s'intéresser à la nature des relations qui lient l'entreprise à ses parties prenantes sans pour autant intégrer la dimension de l'engagement qui occupe pourtant une place de plus en plus importante dans les recherches sur les relations entre l'entreprise et ses parties prenantes.

De nombreuses recherches visent à proposer des principes pour un management de ces parties prenantes (Freeman et McVea, 2001). On peut se demander si le terme de management et le concept qu'il recouvre s'adapte aux relations entre une entreprise et ses parties prenantes. Harrison et St. John (1996) proposent de parler plutôt d'activités de partenariat qui permettent aux entreprises de créer des ponts avec leurs parties prenantes dans la poursuite d'objectifs communs, alors que les techniques traditionnelles de management des parties prenantes facilitent seulement la satisfaction des besoins ou des attentes des parties prenantes. Certaines entreprises vont même au-delà d'un partenariat avec leurs parties prenantes et cherchent à les engager dans un véritable processus d'apprentissage pour la responsabilité globale (Berthoin Antal et Sobczak, 2004).

Cette idée est confortée par les recherches récentes de Greenwood (2007) qui propose de tenir compte de deux dimensions dans l'analyse des pratiques des entreprises à l'égard de leurs parties prenantes : d'une part, la dimension morale comptabilisant le nombre d' " ayant droits " ou de créanciers dont les intérêts sont respectés par l'entreprise ; et, d'autre part, l'engagement des parties prenantes qui mesure la qualité des processus de consultation, de communication et de dialogue permettant aux parties prenantes d'être influentes. Dans cette perspective, l'entreprise doit donc non seulement chercher à satisfaire ses parties prenantes et éviter les conflits d'intérêts, mais également à les engager dans un processus de dialogue permanent et constructif. Selon Maak (2007), les bénéfices d'un tel engagement sont mutuels. Si les parties prenantes voient certaines de leurs attentes satisfaites, l'entreprise bénéficie du capital social que les parties prenantes investissent et qui correspond à " l'ensemble de ressources actuelles ou potentielles qui sont liées à la possession d'un réseau de relations plus ou moins institutionnalisées d'interconnaissance et d'interreconnaissance "(Bourdieu, 1980). L'entreprise peut mobiliser ces ressources pour créer et faire circuler de l'information, de la connaissance et de la confiance au moyen d'une coordination cognitive de parties engagées et contribuer ainsi 


\section{Pour une cartographie des parties prenantes fondée sur leur engagement : une application aux sociétaires d'une banque mutualiste française}

à sa démarche de responsabilité globale. Cet engagement organisationnel peut donc être considéré comme un processus d'apprentissage et être décrit comme le lien entre l'entreprise et ses parties prenantes amenant ceux-ci à croire dans les normes, valeurs et objectifs, à faire des efforts pour son compte et à souhaiter rester en relations avec celle-ci, quel que soit le type de relation.

L'importance de cette notion d'engagement développée dans le champ de la psychologie sociale et cognitive nous amène à la retenir dans la nouvelle cartographie des parties prenantes que nous proposons. Mais fonder la cartographie des parties prenantes sur ce seul engagement organisationnel conduirait à exclure d'autres dimensions. Greenwood précise en effet que l'engagement des parties prenantes ne contribue pas nécessairement à la responsabilité globale dans la mesure où il peut s'agir de parties prenantes « irresponsables », peu concernées par l'environnement économique, social et naturel (Greenwood, 2007).

Prenons l'exemple de l'actionnaire " activiste » pour illustrer nos propos. Selon Logsdon et Van Buren III (2009), cet actionnaire joue un double rôle. En tant que partie prenante primaire, il agit comme un investisseur traditionnel soucieux du retour sur investissement de son capital financier. Son rôle sera uniquement disciplinaire et visera à aligner ses intérêts financiers avec ceux de la direction. Cet engagement privé peut prendre des formes variées telles que des réunions informelles avec la direction, la nomination d'un ou plusieurs de ses représentants au sein du conseil d'administration, l'exercice de ses droits de vote lors de la tenue des assemblées générales des actionnaires ou son adhésion à un club ou à un cercle d'actionnaires, tout en se désintéressant totalement de l'impact social et environnemental des activités de l'entreprise. D'autres actionnaires, au contraire, mobilisent leur capital social pour faire évoluer les pratiques de l'entreprise dans le sens de la responsabilité globale et dans une perspective de long terme. Selon Logsdon et Van Buren III, ils agissent alors en tant que parties prenantes secondaires mobilisant leur capital relationnel (Bhagat, Black et Blair, 2004) qui présente la particularité de combiner l'engagement organisationnel avec d'autres formes d'engagement plus publique (Gillan et Starks, 2007) telles que la mobilisation de réseaux sociaux.

Prenant en compte l'impact de cette dimension, nous proposons une cartographie des parties prenantes fondée sur deux variables liées à l'engagement : l'engagement à l'égard de l'entreprise qualifié d'engagement organisationnel et celui à l'égard des valeurs et principes de la responsabilité globale qualifié d'engagement sociétal. 


\section{2. Le modèle conceptuel des parties plus ou moins engagées}

Selon Healey (1997), l'engagement est un processus d'apprentissage qui se caractérise par la création et la circulation de la confiance, de la connaissance et des valeurs pour fournir un fondement au capital social. L'engagement organisationnel des salariés a fait l'objet de nombreuses recherches en gestion des ressources humaines. La littérature dans ce domaine distingue entre différentes dimensions et différentes cibles de l'engagement. Meyer et Allen (1991, 1997) mettent en évidence trois dimensions de l'engagement organisationnel, celui-ci pouvant être affectif, normatif ou de continuité. L'engagement affectif traduit un attachement émotionnel à l'organisation. Le salarié reste dans l'organisation, car il le désire. L'engagement normatif relève, quant à lui, d'une obligation morale ressentie. Le salarié reste dans l'organisation, car il pense qu'il le devrait. Enfin, l'engagement de continuité - désormais appelé calculé - est lié à la prise de conscience du coût que représenterait le fait de quitter l'organisation. Le salarié reste dans l'organisation, car il en a besoin. L'engagement affectif, normatif et calculé doivent être considérés comme des dimensions différentes de l'engagement plutôt que comme des formes différentes de cet engagement. En effet, ces dimensions ne sont pas exclusives les unes des autres, et l'engagement du salarié peut les comporter toutes à des degrés différents. Un salarié peut ainsi ressentir un fort désir et un grand besoin de rester dans l'organisation (Meyer et Allen, 1991).

L'idée de l'engagement organisationnel peut aussi être transposée à d'autres catégories de parties prenantes, au moins primaires, à savoir les actionnaires, les clients et les fournisseurs. Même si l'engagement organisationnel se traduit différemment pour chaque catégorie, ces acteurs peuvent en effet désirer rester en lien avec l'entreprise, s'y sentir obligés ou le faire par calcul. Cela est vrai en particulier pour les actionnaires pour lesquels on peut distinguer les trois dimensions de l'engagement organisationnel identifiées pour les salariés. Par exemple, dans les entreprises en difficultés financières, l'engagement normatif se manifeste par le soutien des actionnaires qui se sentent « obligés » de garder leurs participations financières (Lemoine et Onnée, 2001). L'engagement affectif implique que l'actionnaire s'identifie à la société dans laquelle il a investi, ce qui peut se manifester par la présence des actionnaires à des comités consultatifs, à des clubs ou des cercles d'actionnaires, à des séminaires de formation de bourse, à des visites des sites d'exploitation ou à des expositions culturelles. Cependant, soulignons que l'engagement affectif et normatif ne naissent en principe que si l'entreprise garantit par ailleurs l'engagement calculé correspondant à des résultats financiers acceptables.

Les trois dimensions révélées dans le domaine de l'engagement organisationnel peuvent être transposées pour l'engagement sociétal. Les activités visant à favoriser une prise en compte à la fois des aspects économiques, sociaux et environnementaux peut en effet s'expliquer par un attachement affectif à ces 


\section{Pour une cartographie des parties prenantes fondée sur leur engagement : une application aux sociétaires d'une banque mutualiste française}

valeurs, par un sentiment d'obligation morale ou par un raisonnement calculé. Les parties prenantes d'une organisation peuvent ainsi soutenir une politique de responsabilité globale parce qu'elles le désirent, parce qu'elles s'y sentent obligées ou parce qu'elles pensent que c'est nécessaire.

En évaluant les deux types d'engagement pour les différentes parties prenantes d'une entreprise, nous pouvons établir une nouvelle cartographie de celles$\mathrm{ci}$, fondée sur une répartition selon deux axes, un premier axe exprimant l'engagement organisationnel, le second l'engagement sociétal (figure $n^{\circ} 1$ ). Cette cartographie fait apparaître quatre profils parmi l'ensemble des parties prenantes d'une entreprise. Un premier groupe se caractérise à la fois par un faible degré d'engagement organisationnel et un faible degré d'engagement des parties prenantes. Qualifié de « parties passives », les membres de ce groupe de parties prenantes ne pourront que difficilement être impliqués dans une démarche de responsabilité globale sans autre effort de la part des dirigeants. Un deuxième groupe combine au contraire un fort degré d'engagement organisationnel et un fort degré d'engagement sociétal. Les membres de ce groupe que nous qualifions de " parties engagées » constituent pour les dirigeants de l'entreprise des vecteurs importants, voire des moteurs, de leur démarche de responsabilité globale. Les deux autres groupes présentent un déséquilibre entre les deux types d'engagement. Les " parties alliées » ont un fort degré d'engagement organisationnel, mais un faible degré d'engagement sociétal, alors que c'est l'inverse pour les « parties militantes ».

Figure $\mathrm{n}^{\circ} 1:$ une nouvelle cartographie des parties engagées

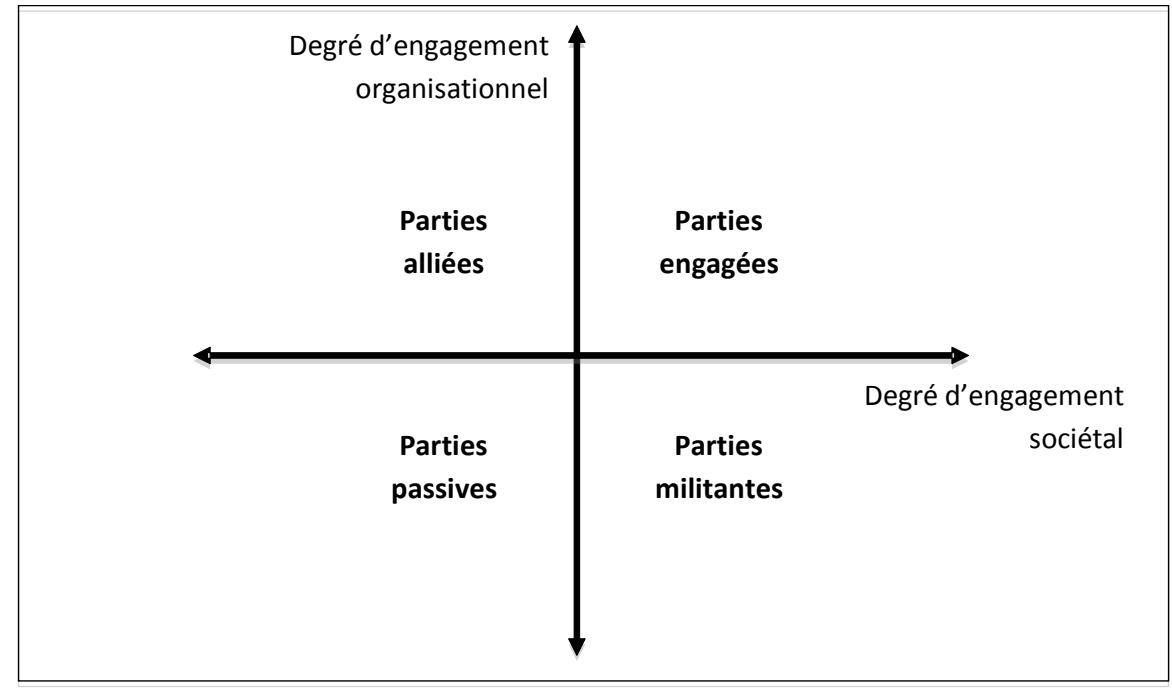


Bien évidemment, l'engagement organisationnel et sociétal peuvent évoluer, nécessitant donc une approche dynamique de cette cartographie. Son actualisation régulière permettra ainsi de mesurer les évolutions dans l'engagement des parties prenantes et d'évaluer, le cas échéant, l'impact des politiques managériales mises en œuvre par les dirigeants de l'organisation, à la fois sur l'engagement organisationnel et sur l'engagement sociétal, l'objectif pouvant être de faire évoluer les membres des différents groupes vers la catégorie des « parties engagées ».

\section{Une cartographie des sociétaires d'une banque mutualiste française}

Afin de tester cette nouvelle cartographie des parties prenantes, nous avons mené une première étude auprès d'une catégorie particulière de parties prenantes d'une entreprise elle-même particulière : les sociétaires de la Banque Populaire Atlantique (désormais BPA). Les sociétaires d'une banque mutualiste sont une catégorie de partie prenante intéressante à étudier pour tester notre modèle conceptuel, car par définition leur statut juridique est double, voire triple. Ils sont à la fois des actionnaires et des clients de la banque, et peuvent parfois en être des salariés. Contrairement aux actionnaires d'une banque cotée, ils n'ont aucun droit aux réserves et ne perçoivent aucun "dividende financier » mais un « dividende coopératif $»^{56}$ dont une partie est versée pour soutenir les démarches de responsabilité globale. Les sociétaires peuvent donc non seulement correspondre à trois types de parties prenantes primaires (actionnaires, clients, voire salariés), mais jouer également le rôle d'une partie prenante secondaire militant pour défendre une cause sociale et/ou environnementale. Pour analyser les intérêts et les attentes des sociétaires, il semble donc important de créer une classification plus fine comme celle que nous proposons avec la cartographie fondée sur l'engagement organisationnel et sociétal.

LaBPAest engagée depuis plusieurs années dans une démarche de responsabilité globale qui se traduit notamment par l'intégration des enjeux économiques, sociaux et environnementaux dans son plan stratégique de moyen terme. Pour continuer à améliorer leur démarche, les dirigeants de cette banque souhaitent développer l'engagement de leurs parties prenantes et notamment des sociétaires. Afin de déterminer les actions à mener dans cette perspective, nous avons mené une étude qualitative visant dans un premier temps à cartographier les différents sociétaires de la BPA, en fonction de leur engagement organisationnel et sociétal.

L'intérêt de cette étude dépasse le cadre de la BPA et apporte une contribution aux questions que les managers des banques mutualistes se posent sur

56. Cf. étude « 60 millions de sociétaires des banques coopératives. Quels enseignements ? » réalisée en 2006 par le regroupement européen des banques coopératives. 


\section{Pour une cartographie des parties prenantes fondée sur leur engagement: une application aux sociétaires d'une banque mutualiste française}

la manière de renouveler les relations avec leurs sociétaires et de mieux les engager dans le développement des banques. D'une manière générale, les banques mutualistes françaises font en effet face à un affaiblissement de leur sociétariat, ce qui se traduit à la fois par une réduction du nombre de sociétaires et par une moindre implication dans la gouvernance, toutes deux liées à des évolutions sociétales, réglementaires et concurrentielles (Richez-Battesti, 2006). A l'origine, les sociétaires étaient caractérisés par une grande homogénéité socioprofessionnelle conduisant à un certain engagement corporatiste et des intérêts communs. Aujourd'hui, les sociétaires de ces banques regroupent des catégories socioprofessionnelles très différentes et ils sont au mieux réunis par un certain nombre de valeurs communes. Les initiatives de communication de la part des banques mutualistes sur leurs démarches de responsabilité globale et les racines que celles-ci trouvent dans les valeurs mutualistes et corporatives (Chifflet, 2006) visent d'ailleurs à renforcer ce socle de valeurs communes. Néanmoins, cette communication se révèle souvent insuffisante et peut générer de nouveaux risques liés à des questions sur la légitimité de leur gouvernance. De ce fait, l'un des enjeux actuels pour les banques coopératives est d'augmenter très fortement le nombre de sociétaires et de renforcer leur engagement en les faisant évoluer d'un statut passif à une participation plus active.

\subsection{La méthodologie}

Pour opérationnaliser notre modèle de cartographie des parties prenantes, nous avons décidé de soumettre un questionnaire en ligne à un échantillon des sociétaires de la BPA. L'objectif était de créer une échelle de l'engagement organisationnel et sociétal des parties prenantes. Pour l'engagement organisationnel, nous avons choisi de partir de l'échelle de Meyer et Allen (1991) mesurant les trois dimensions de l'engagement organisationnel des salariés pour l'adapter aux sociétaires de BPA. Pour l'engagement sociétal, nous avons interrogé les sociétaires sur des comportements traduisant la prise en compte des enjeux sociaux et environnementaux. Le questionnaire a été construit en plusieurs étapes. Dans une première étape, nous avons réalisé deux focus groupes, en décembre 2007 et en janvier 2008, réunissant à chaque fois une vingtaine de sociétaires de catégories socioprofessionnelles et d'âges différents. Dans une seconde étape, nous avons testé notre questionnaire auprès de 72 étudiants ayant choisi de suivre des cours spécifiques sur la responsabilité globale dans une école de management.

Le questionnaire présenté en annexe se compose de trois parties. Dans la partie 1 intitulée « Vous, en tant que citoyen » (Questions 1 à 10), 10 items mesurent l'engagement sociétal des sociétaires. La partie 2 appelée « Vous et La Banque Populaire Atlantique » (Questions 11 à 27) mesure l'engagement organisationnel des sociétaires envers la BPA. De la question 11 à 16 , on retrouve les items de Meyer et Allen pour mesurer l'engagement affectif. Les questions 17 à 19 et 26 
à 27 mesurent la dimension de l'engagement normatif. Enfin, les questions 20 à 25 mesurent la dernière dimension : celle de l'engagement calculé. La partie 3 correspond à la fiche signalétique du répondant.

Ce questionnaire a été rempli via le site : http://freonlinesurveys.com indiqué par courriel aux 2000 sociétaires internautes sur les 100022 sociétaires de BPA. Au total, 382 questionnaires ont été totalement remplis (soit un taux de réponse $17,36 \%$ ).

Le profil des répondants précisé dans l'encadré 1 est assez proche de celui de l'ensemble des sociétaires de la BPA.

Encadré 1 : Les caractéristiques des sociétaires interrogés

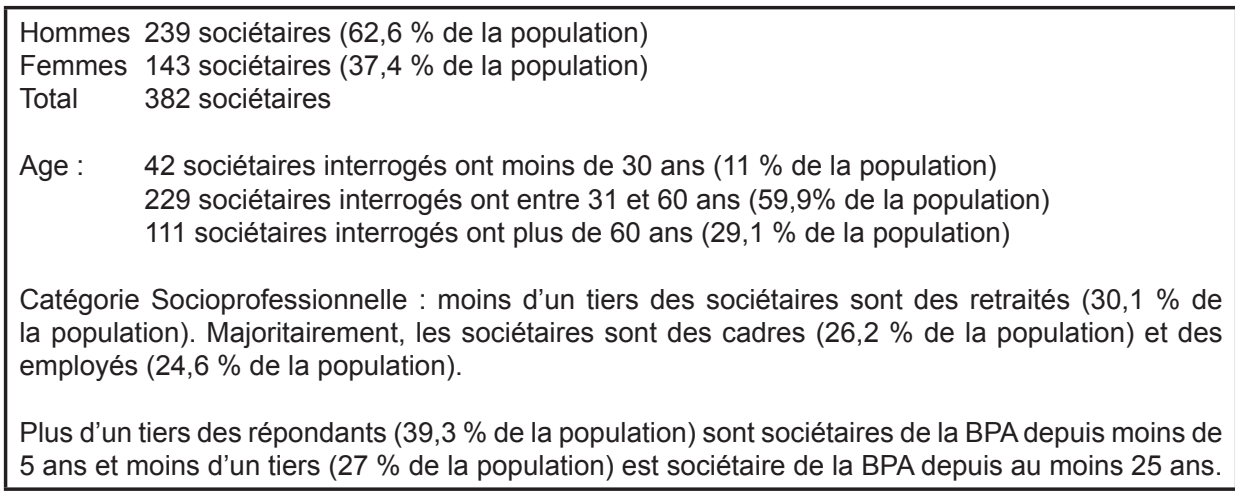

\subsection{Les résultats}

Globalement, les résultats de l'enquête montrent un engagement organisationnel relativement faible des sociétaires de la BPA. Ainsi, 287 sociétaires sur $382(75,1$ $\%$ ) ont répondu qu'ils ne participaient pas aux assemblées générales de BPA. Ces résultats ne sont pas spécifiques à la BPA, d'autres études ayant montré que le taux de participation des sociétaires aux assemblées générales des sociétés mutualistes et coopératives se situe entre 10 et 30 \% (Sibieude et Vidal, 2008). L'étude met en revanche en évidence l'engagement sociétal des sociétaires. Ainsi, 133 des sociétaires $(34,82 \%$ de la population) militent dans des associations ou s'engagent dans la vie publique. Certes, ce taux correspond au taux d'adhésion des français aux associations (34 \% : INSEE ${ }^{57}$ ), mais l'engagement que nous mesurons ne porte que sur l'engagement dans les associations humanitaires, caritatives ou environnementales, alors que le taux d'adhésion calculé inclut aussi les français qui s'engagent dans d'autres associations, notamment sportives (14\% de la population française). L'engagement sociétal des sociétaires de la BPA se manifeste aussi par leur intérêt pour l'investissement responsable. En effet, 105 des sociétaires (27,49\% de la population) déclarent tenir compte des

57. Taux d'adhésion à différentes associations [en ligne]. 2006. Disponible sur le site de l'INSEE : <http://www.insee.fr/fr/themes/ tableau.asp?reg_id=0\&ref_id=NATSOS05512\%C2> (consulté le 10 novembre 2009). 


\section{Pour une cartographie des parties prenantes fondée sur leur engagement : une application aux sociétaires d'une banque mutualiste française}

critères sociaux et environnementaux dans le choix de leur épargne et leurs investissements.

Au-delà de ces résultats globaux, notre objectif était de classer les sociétaires de la BPA en fonction de leur engagement organisationnel et sociétal. A cette fin, les 27 premiers items ont d'abord fait l'objet d'une analyse en composantes principales (désormais ACP) pour identifier des variables composites indépendantes. L'objectif était de vérifier si parmi ces variables, on retrouvait bien les deux variables d'engagement organisationnel et sociétal. Cette analyse sur les 27 variables initialement choisies a permis de dégager 7 facteurs présentés dans le tableau $\mathrm{n}^{\circ} 1$. Ces facteurs représentent $56,39 \%$ de l'information avec un $\mathrm{KMO}$ de 0,842 .

Tableau $n^{\circ} 1$ : Le tableau de l'analyse des composantes portant sur les items de l'engament organisationnel et sociétal

\begin{tabular}{|c|c|c|}
\hline Facteurs & Attributs & $\begin{array}{l}\text { Coefficient de } \\
\text { corrélation linéaire }\end{array}$ \\
\hline $\begin{array}{l}\text { Engagement } \\
\text { organisationnel }\end{array}$ & $\begin{array}{l}\text { Fierté de dire aux autres que je suis sociétaire de la BPA } \\
\text { Fidèle à BPA car beaucoup investi(e) } \\
\text { Fidèle à la BPA car elle possède les mêmes valeurs } \\
\text { Heureux d'être sociétaire } \\
\text { Plaisir à discuter de la Banque Populaire (désormais BPA) } \\
\text { Concerné(e) par les problèmes de BPA } \\
\text { Même si désavantagé(e), je resterai sociétaire de BPA } \\
\text { Difficile de quitter BPA même si je le souhaitais } \\
\text { Culpabilité si je devais quitter la BPA } \\
\text { Je suis resté(e) à BPA car peu d'opportunités se sont présentées } \\
\text { Aucun attachement à BPA } \\
\text { Ne se sent pas sociétaire de BPA } \\
\text { Facile de quitter BPA car je ne suis pas trop impliquée } \\
\text { Indifférent entre être sociétaire de BPA ou d'une autre banque }\end{array}$ & $\begin{array}{l}0,704 \\
0,682 \\
0,682 \\
0,670 \\
0,639 \\
0,591 \\
0,579 \\
0,550 \\
0,529 \\
0.480 \\
-0,518 \\
-0,503 \\
-0,423 \\
-0,403\end{array}$ \\
\hline $\begin{array}{l}\text { Engagement } \\
\text { sociétal }\end{array}$ & $\begin{array}{l}\text { Agriculture biologique } \\
\text { Commerce équitable } \\
\text { Produits régionaux } \\
\text { Dons } \\
\text { Fruits/légumes de saison } \\
\text { Militant(e) dans une association et/ou dans la vie publique } \\
\text { Critères sociaux et environnementaux pris en compte dans les } \\
\text { placements financiers }\end{array}$ & $\begin{array}{l}0,656 \\
0,625 \\
0,569 \\
0,566 \\
0,499 \\
0,457 \\
0,457\end{array}$ \\
\hline $\begin{array}{l}\text { Manque } \\
\text { d'engagement } \\
\text { affectif }\end{array}$ & $\begin{array}{l}\text { Envisage de quitter BPA car peu impliqué(e) } \\
\text { Aucune obligation à rester sociétaire de BPA }\end{array}$ & $\begin{array}{l}0,512 \\
0,461\end{array}$ \\
\hline $\begin{array}{l}\text { Manque } \\
\text { d'engagement } \\
\text { normatif }\end{array}$ & $\begin{array}{l}\text { Si c'était à mon désavantage, je resterai sociétaire } \\
\text { Culpabilité si je devais quitter BPA }\end{array}$ & $\begin{array}{l}-0,457 \\
-0,511\end{array}$ \\
\hline $\begin{array}{l}\text { Engagement } \\
\text { calculé }\end{array}$ & $\begin{array}{l}\text { Trop coûteux de quitter BPA } \\
\text { Préoccupé(e) par les pertes liées à mes investissements }\end{array}$ & $\begin{array}{l}0,647 \\
0,413\end{array}$ \\
\hline $\begin{array}{l}\text { Manque } \\
\text { d'engagement } \\
\text { sociétal }\end{array}$ & $\begin{array}{l}\text { Transports publics ou covoiturage } \\
\text { Militant(e) dans une association et/ou dans la vie publique }\end{array}$ & $\begin{array}{l}-0,463 \\
-0,415\end{array}$ \\
\hline $\begin{array}{l}\text { Engagement } \\
\text { environnemental }\end{array}$ & $\begin{array}{l}\text { Tri des déchets } \\
\text { Transports publics ou covoiturage }\end{array}$ & $\begin{array}{l}0,556 \\
0,439\end{array}$ \\
\hline
\end{tabular}


Le premier facteur identifié par l'ACP correspond à l'engagement organisationnel. II représente $21,57 \%$ de l'information totale et regroupe 15 des 18 items couvrant les trois dimensions de l'engagement organisationnel. En premier, figurent plusieurs items mesurant l'engagement normatif : item $\mathrm{n}^{\circ} 18$ " fierté de dire aux autres que je suis sociétaire de BPA » $(0,704)$ dans notre questionnaire (annexe 1), puis l'item $n^{\circ} 23$ « fidèle à la BPA car beaucoup investi(e) » tant sur le plan économique, émotionnel ou social, enfin l'item $\mathrm{n}^{\circ} 17$ « fidèle à la BPA car elle possède les mêmes valeurs » $(0,682)$. Ensuite, on trouve un premier item (le numéro 11 dans le questionnaire) qui mesure l'une des dimensions de l'engagement affectif celui d'être « heureux d'être sociétaire » $(0,670)$. A l'inverse, on constate que le manque d'engagement affectif est corrélé négativement à cet ACP. Ainsi, l'item $\mathrm{n}^{\circ} 14$ « indifférent entre être sociétaire de la BPA ou d'une autre banque » $(-0,403)$, l'item $n^{\circ} 15$ « ne se sent pas sociétaire » $(-0,503)$ et l'item $n^{\circ} 16$ « aucun attachement à la BPA » $(-0,518)$ sont corrélés négativement à cet $\mathrm{ACP}$. On constate également que quelques items mesurant la dimension calculée de l'engagement sont corrélés positivement ou négativement à cet ACP. C'est notamment le cas de l'item $n^{\circ} 20$ « difficile de quitter BPA même si je le souhaitais » $(0,550)$.

Le deuxième facteur qui représente 10,23 \% de l'information totale correspond à l'engagement sociétal. En effet, cet ACP est composé de 7 des 11 items mesurant l'engagement sociétal des sociétaires. Ceux qui sont les plus fortement corrélés à cet axe sont l'item $n^{\circ} 2$ « agriculture biologique » $(0,656)$, l'item $n^{\circ}$ 1 « commerce équitable » $(0,625)$, l'item $n^{\circ} 4$ « produits régionaux » $(0,569)$ et l'item $n^{\circ} 7$ « dons » $(0,566)$. On constate que cet axe est faiblement corrélé positivement aux deux autres items environnementaux que l'on retrouve dans le dernier axe factoriel. Ce résultat est conforme à une étude récente de Sibieude et Vidal (2008) qui indique que l'une des caractéristiques du sociétariat en France est son engagement sociétal à partir de l'ancrage régional du groupe mutualiste ou coopératif.

Les trois facteurs suivants sont composés d'une des trois dimensions de l'engagement organisationnel non constitutives du premier ACP. De manière cumulée, ces trois axes représentent 16,64\% de l'information totale. Le troisième facteur représente $6,36 \%$ de l'information totale et mesure le « manque d'engagement affectif ». L'item $n^{\circ} 21$ " envisage de quitter la BPA car peu impliqué(e) » $(0,512)$ est corrélée positivement à ce facteur. Le quatrième facteur représente $5,32 \%$ de l'information totale et mesure quelques dimensions du manque d'engagement normatif. L'item $n^{\circ} 27$ « culpabilité si je devais quitter la BPA » $(-0,511)$ et l'item $n^{\circ} 19$ "préoccupé(e) par les pertes liées à mes investissements " sont corrélées négativement à ce facteur. Nous qualifierons donc ce facteur de manque d'engagement normatif. 


\section{Pour une cartographie des parties prenantes fondée sur leur engagement : une application aux sociétaires d'une banque mutualiste française}

Enfin, les deux derniers facteurs sont des facteurs représentant deux dimensions de l'engagement sociétal non prises en compte dans le deuxième facteur. Le cinquième facteur représente seulement $4,20 \%$ de l'information totale et est faiblement corrélé négativement à deux items : I'un mesurant l'engagement environnemental du sociétaire par l'utilisation des " transports publics ou covoiturage " (item $n^{\circ} 6$ avec un coefficient de corrélation de -0,463) et le second mesurant l'attitude militante de ce même sociétaire par une participation active dans une association ou dans la vie publique (item $n^{\circ} 8$ avec un coefficient de corrélation linéaire de $-0,415)$. Sur cet axe, on mesure le « manque d'engagement sociétal ». Le sixième facteur représente $3,75 \%$ de l'information totale. Al'inverse du précédent, les enjeux environnementaux sont corrélés positivement à ce facteur. Ainsi, l'item $\mathrm{n}^{\circ} 5$ « tri des déchets " est corrélé positivement à cet axe $(0,556)$; il en est de même l'item $n^{\circ} 6$ " transports publics ou covoiturage " $(0,439)$. Sur cet axe, on mesure l'une des dimensions de l'engagement sociétal que nous qualifierons d'engagement environnemental.

Dans un deuxième temps, à partir des scores factoriels, une cartographie des sociétaires de la BPA a été construite par la méthode de classification par « nuées dynamiques ». Cette méthode permet de mettre en évidence 4 groupes d'effectifs relativement homogènes. Le tableau $n^{\circ} 2$ présente les moyennes des 7 facteurs par classes de sociétaires.

Tableau $\mathrm{n}^{\circ} 2$ : Les moyennes des dimensions des caractéristiques du sociétariat de BPA

\begin{tabular}{|c|c|c|c|c|}
\hline $\begin{array}{l}4 \text { groupes de sociétaires } \\
\text { Nombre de sociétaires par groupe }\end{array}$ & $\begin{array}{c}1 \\
N=93\end{array}$ & $\begin{array}{c}2 \\
N=102\end{array}$ & $\begin{array}{c}3 \\
N=126\end{array}$ & $\begin{array}{c}4 \\
N=60\end{array}$ \\
\hline Engagement organisationnel & 0,04908 & $-0,82461$ & 0,61896 & 0,02596 \\
\hline Engagement sociétal & $-0,48809$ & 0,82846 & 0,02679 & $-0,70811$ \\
\hline Pas d'engagement affectif & 0,55705 & $-0,35355$ & $-0,14186$ & 0,03550 \\
\hline Pas d'engagement normatif & $-0,64030$ & $-0,17882$ & 0,62670 & $-0,01959$ \\
\hline Engagement calculé & 0,75326 & $-0,05595$ & $-0,22437$ & $-0,60126$ \\
\hline Pas d'engagement sociétal & $-0,32081$ & 0,13810 & 0,09060 & 0,07224 \\
\hline Engagement environnemental & 0,36424 & $-0,07594$ & 0,39445 & $-1,26382$ \\
\hline
\end{tabular}

Les sociétaires du groupe 1 (au nombre de 93 , soit $24,35 \%$ des répondants) ne sont engagés ni à l'égard de la BPA, ni à l'égard des principes de la responsabilité globale. Ces sociétaires sont uniquement caractérisés par leur engagement calculé $(0,75)$, lié à la prise de conscience du coût que représenterait le fait de quitter la BPA. II existe néanmoins un engagement normatif $(-0,64)$ mais pas d'engagement affectif $(0,56)$. En effet, l'engagement affectif implique une forte identification du sociétaire à l'organisation. Or, ici le devoir de rester sociétaire de BPA semble davantage motivé par les engagements financiers contractés avec la BPA. On qualifiera donc ce groupe de sociétaires de "sociétaires passifs ». Les sociétaires du groupe 2 (au nombre de 102, soit $26,70 \%$ des répondants) s'engagent fortement à l'égard de la responsabilité globale $(0,83)$, mais sont négativement engagés dans l'organisation $(-0,82)$. Conformément au modèle 
conceptuel présenté dans la première section (tableau $\mathrm{n}^{\circ} 1$ ), nous qualifierons ce groupe de sociétaires de " sociétaires militants ».

Les sociétaires du groupe 3 (au nombre de 126 soit un tiers des répondants) montrent un fort degré d'engagement organisationnel $(0,82)$ mais ne ressentent pas le devoir de rester sociétaire si c'est à leur désavantage $(0,63)$. De ce fait, on ne peut pas qualifier ces sociétaires de "parties alliées » car le risque de retrait subsiste. Nous qualifierons donc ce groupe de " sociétaires participatifs ».

Les actionnaires du groupe 4 (au nombre de 60 soit 15,71 \% des répondants) montrent un degré d'engagement sociétal négatif $(-0,71)$ et un degré d'engagement calculé négatif $(-0,60)$. Ce groupe de sociétaires n'est ni concerné par les préoccupations sociétales ni par les résultats de ses placements ou investissements réalisés au sein de la BPA. Nous qualifierons donc ce groupe de sociétaires de " sociétaires atones».

Conformément aux études antérieures portant sur le sociétariat d'autres banques mutualistes, on constate donc que le sociétariat de la BPA est éclaté et constitué d'une pluralité de parties plus ou moins engagées, voire atones (Figure $n^{\circ} 2$ ).

Figure $\mathrm{n}^{\circ} 2$ : La cartographie des sociétaires de la BPA, le 30 décembre 2008

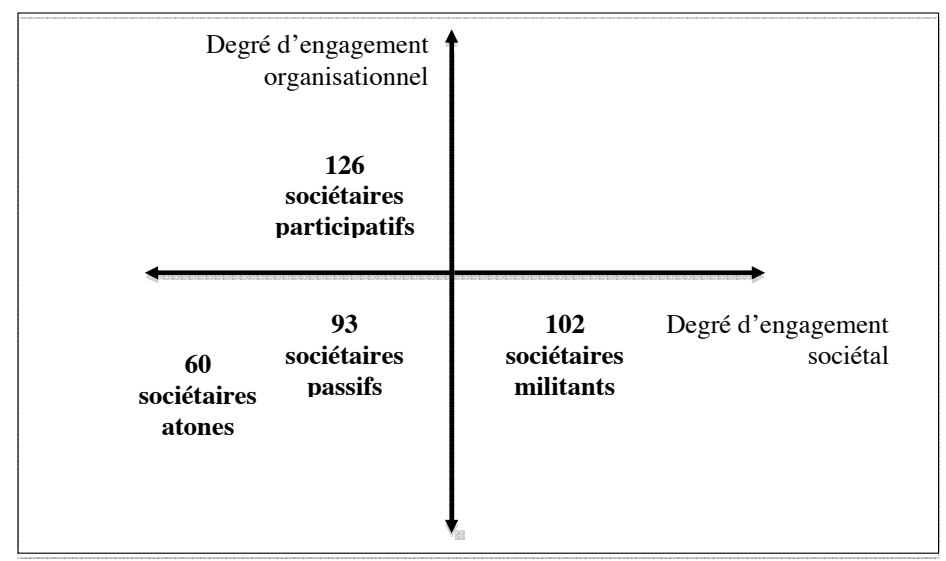

Cette cartographie des sociétaires de la BPA peut permettre aux dirigeants de cette banque de développer une communication et des initiatives adaptées à chacune de ces catégories visant à renforcer leur engagement organisationnel et sociétal, tout en gardant évidemment une cohérence d'ensemble. La banque pourrait ainsi développer une communication spécifique pour les sociétaires militants engagés à l'égard de la responsabilité globale, mais peu impliqués dans la BPA, pour mieux les informer sur les différentes actions qu'elle a mis en place 


\section{Pour une cartographie des parties prenantes fondée sur leur engagement : une application aux sociétaires d'une banque mutualiste française}

dans ce domaine. Pour les sociétaires participatifs qui se caractérisent par leur engagement organisationnel à l'égard de la BPA sans faire état d'un engagement sociétal particulier, la communication pourrait au contraire mettre l'accent sur la nécessité pour les banques de mener des actions dans le domaine de la responsabilité globale et sur l'impact économique positif d'un tel engagement.

\section{Conclusion}

L'intérêt de cet article réside dans le fait qu'il propose une nouvelle cartographie des parties prenantes fondées sur leur engagement organisationnel et sociétal et qu'il la teste pour une catégorie particulière, les sociétaires d'une banque mutualiste. II peut ainsi contribuer à mieux comprendre la diversité des intérêts et attentes des sociétaires, qu'il est difficile de classer dans les cartographies habituelles des parties prenantes et sur lesquels il existe assez peu de recherches en gestion. Pour aller plus loin, il convient d'analyser en profondeur les caractéristiques des membres des différents groupes crées par la méthode de classement par nuées dynamiques, concernant leur sexe, leur âge, leur catégorie socioprofessionnelle et leur ancienneté comme sociétaire de la BPA.

Par ailleurs, pour mesurer tout l'intérêt de cette nouvelle cartographie, il s'agit de reconduire régulièrement le même questionnaire auprès de la même population ou d'un échantillon plus réduit mais représentatif, afin d'observer les évolutions dans l'engagement des sociétaires, en tenant compte de l'impact des événements externes et des pratiques managériales mises en place. Par ailleurs, il convient de soumettre le questionnaire aux autres catégories de parties prenantes de la BPA, notamment aux salariés et aux clients qui ne sont pas sociétaires, pour évaluer si l'on retrouve un éclatement similaire au sein de ces catégories. Cela permettrait de vérifier si, en appliquant la méthode de classement par nuées dynamiques aux réponses données par toutes ces parties prenantes, les groupes ainsi créés sont composés de membres des différentes catégories de parties prenantes ou s'ils sont spécifiques à chaque catégorie de partie prenantes indiquant alors que le degré d'engagement organisationnel et sociétal diffère d'une manière trop importante entre les sociétaires, les salariés et les clients.

\section{Bibliographie}

Berthoin Antal A. \& Sobczak A. (2004), « Beyond CSR: Organisational learning for global responsibility ", Journal of General Management, Vol. 30, No 2, p. 77-98.

Bhagat S., Black B. \& Blair M. (2004), "Relational investing and firm performance ", Journal of Financial Research, Vol. 27, № 1, p. 1-30.

Bourdieu P. (1980), « Le capital social », Actes de la Recherche en Sciences Sociales, Vol. 31, No 1, p. 2-3.

Caroll A. B. (1989), Business \& Society, South Western Publishing, Cincinnati.

Chiffet J.-P. (2006), " Que signifie avoir 60 millions de sociétaires ? ", Communication à la Convention des banques coopératives européennes, 28 mars.

Donaldson T. \& Preston L. E. (1995), « The stakeholder theory of the corporation: concepts, 
evidence, and implications ", Academy of Management Review, Vol. 20, No 1, p. 85-91. Freeman R. E. (1984). Strategic management: a stakeholder approach, Pitman/Ballinger, Boston.

Freeman R. E. \& Gilbert D. R. (1987), « Managing stakeholder relationships », in Sethi S. P. \& C. M. Falbe (Eds.), Business and Society: Dimensions of Conflict and Cooperation, Heath, Lexington p. 397-423.

Freeman R.E. \& Mc Vea J. (2001), «A stakeholder approach to strategic management », in Hitt, M., E. Freeman \& J. Harrison (Eds.), Handbook of Strategic Management, Blackwell Publishing, Oxford.

Frooman J. (1999), "Stakeholder influence strategies », Academy of Management Review, Vol. 24, No 2, p. 1991-205.

Gillan, S. \& L. Starks (2007), « The evolution of shareholder activism in the United States », Journal of Applied Corporate Finance, Vol. 19, No 1, p. 55-73.

Greenwood M. (2007), « Stakeholder engagement: Beyond the myth of Corporate Responsibility ", Journal of Business Ethics, Vol. 74, p. 315-327.

Harrison J. S. \& St. John C. H. (1996), « Managing and partnering with external stakeholders ", Academy of Management Executive, Vol. 10, No 2, p. 46-60.

Lemoine J.-F. \& Onnée S. (2001), "Vers une meilleure compréhension du statut "d'actionnaire individuel-client », Gestion 2000, No 4, p. 127-138.

Logsdon J. M. \& Van Buren III H. J. (2009), « Beyond the proxy vote: Dialogues between shareholder activists and corporations ", Journal of Business Ethics, Vol. 87, p. 353-365.

Maak T. (2007), « Responsible leadersip, stakeholder engagement, and the emergence of social capital », Journal of Business Ethics, Vol. 74, p. 329-343.

Mercier S. (2006), « La théorie des parties prenantes : une synthèse de la littérature », in $\mathrm{M}$. Bonnafous-Boucher \& Y. Pesqueux (dir.), Décider avec les parties prenantes. Approches d'une nouvelle théorie de la société civile, La Découverte, p. 157-172.

Meyer J. P. \& Allen N. J. (1997), Commitment in the workplace: Theory, research and application, Sage, Thousand Oaks.

Meyer J. P. \& Allen N. J. (1991), «A three-component conceptualization of Organizational commitment », Human Resource Management Review, Vol. 1, No 1, p. 61-.89.

Mitchell R. K., Agle B. R. \& Wood D. J. (1997), " Towards a theory of stakeholder identification and salience: Defining the principle of who and what really counts ", Academy of Management Review, Vol. 22, No 4, p. 853-886.

Post J. E., Preston L. E. \& Sachs S. (2002), « Managing the extended enterprise: The new stakeholder view », California Management Review, Vol. 45, No 1, p. 6-28.

Richez-Battesti N. (2006), "Gouvernance coopérative et reconquête du sociétariat : une aventure inachevée ? Le cas des banques coopératives en France », communication à la XXIlème conférence internationale de recherche coopérative.

Scholes E. \& Clutterbuck D. (1998), « Communication with stakeholders: An integrated approach », Long Range Planning, Vol. 31, No 2, p. 227-238.

Sibieude T. et Vidal R. (2008) «Enjeux et perspectives du sociétariat des groupes mutualistes complexes face aux stratégies de développement à l'échelle du groupe : quelques enseignements du cas du Groupe MACIF », working paper DR 08011, ESSEC Centre de Recherche, Cergy.

Wolfe R. A. \& Putler D. S. (2002). « How tight are the ties that bind stakeholder groups », Organization Science, Vol. 13, No 1, p. 64-80.

Wood D. J. (1994), Business and Society, Harper Collins, New York. 


\section{Pour une cartographie des parties prenantes fondée sur leur engagement: une application aux sociétaires d'une banque mutualiste française}

\section{Annexe 1 : Le questionnaire}

\section{Partie $\mathrm{n}^{\circ} 1$ : « Vous, en tant que citoyen »}

Veuillez indiquer, en entourant un chiffre de 1 à 5, à quelle fréquence vous adoptez les comportements suivants :
(1) Jamais
(2) Rarement
(3) Parfois
(4) Souvent
(5) A chaque fois que c'est possible

1. J'achète les produits issus du commerce équitable.

2. J'achète les produits issus de l'agriculture biologique.

3. J'achète les fruits et légumes de saison.

4. J'achète les produits régionaux

5. Je trie mes déchets.

6. Je privilégie les transports publics ou le co- voiturage.

7. Je fais des dons à des associations humanitaires, caritatives ou environnementales.

8. Je milite dans des associations ou je m'engage dans la vie publique.

9. Je tiens compte de critères sociaux et environnementaux dans le placement de mon épargne ou le choix de mes investissements financiers.

10. Je me renseigne sur l'actualité du développement durable en lisant des revues, en consultant Internet ou en participant à des réunions.

Partie $n^{\circ} 2$ : « Vous et la Banque Populaire Atlantique »

Veuillez indiquer, en entourant un chiffre de 1 à 5, dans quelle mesure vous êtes en accord avec les affirmations suivantes:
(1) Pas du tout d'accord
(2) Plutôt d'accord
(3) Moyennement d'accord
(4) Plutôt d'accord
(5) Tout à fait d'accord

11. Je suis très heureux(se) d'être sociétaire de la Banque Populaire.

12. J'ai plaisir à discuter de la Banque Populaire à mes collègues, mes amis, à ma famille.

13. Je me sens vraiment concerné(e) par les problèmes de la Banque Populaire.

14. J'aurais pu tout aussi bien être sociétaire ou actionnaire d'une autre banque.

15. Je ne me sens pas sociétaire de la Banque Populaire

\begin{tabular}{|l|l|l|l|l|l}
1 & 2 & 3 & 4 & 5 \\
\hline 1 & 2 & 3 & 4 & 5 \\
\hdashline 1 & 2 & 3 & 4 & 5 \\
\hdashline 1 & 2 & 3 & 4 & 5 \\
\hdashline 1 & 2 & 3 & 4 & 5
\end{tabular}


16. Je ne suis pas attaché(e) à la Banque Populaire

17. Je suis fidèle à la Banque Populaire car elle possède les mêmes valeurs que les miennes.

18. Je suis fier(e) de dire aux autres que je suis sociétaire de la Banque Populaire.

19. Je suis préoccupé(e) par les pertes liées à mes investissements réalisés chez la Banque Populaire.

20. II me serait difficile de quitter la Banque Populaire aujourd'hui, même si je le souhaitais.

21. Je peux envisager de quitter la Banque Populaire, étant donné que je ne me suis pas trop impliqué(e) en tant que sociétaire.

22. Ce serait trop coûteux pour moi de quitter la Banque Populaire aujourd'hui.

23. Je suis fidèle à la Banque Populaire, car j'ai beaucoup investi dans cette banque que ce soit économiquement, émotionnellement et socialement.

24. Je n'ai jamais envisagé de quitter la Banque Populaire, car peu d'opportunités se sont présentées à moi.

25. Je considère que je n'ai aucune obligation à rester sociétaire de la Banque Populaire.

26. Même si c'était à mon désavantage, je resterai sociétaire de la Banque Populaire.

27. Je me sentirai coupable si je devais quitter la Banque Populaire.

\begin{tabular}{|l:l:l:l:l}
1 & 2 & 3 & 4 & 5 \\
\hline 1 & 2 & 3 & 4 & 5 \\
\hline 1 & 2 & 3 & 4 & 5 \\
\hline 1 & 2 & 3 & 4 & 5 \\
\hdashline 1 & 2 & 3 & 4 & 5 \\
\hdashline 1 & 2 & 3 & 4 & 5 \\
\hdashline 1 & 2 & 3 & 4 & 5 \\
\hdashline 1 & 2 & 3 & 4 & 5 \\
\hdashline 1 & 2 & 3 & 4 & 5 \\
\hdashline 1 & 2 & 3 & 4 & 5 \\
\hdashline 1 & 2 & 3 & 4 & 5
\end{tabular}


Copyright of Revue Management et Avenir is the property of Management Prospective Editions and its content may not be copied or emailed to multiple sites or posted to a listserv without the copyright holder's express written permission. However, users may print, download, or email articles for individual use. 
Copyright of Revue Management et Avenir is the property of Management Prospective Editions and its content may not be copied or emailed to multiple sites or posted to a listserv without the copyright holder's express written permission. However, users may print, download, or email articles for individual use. 
Copyright of Revue Management et Avenir is the property of Management Prospective Editions and its content may not be copied or emailed to multiple sites or posted to a listserv without the copyright holder's express written permission. However, users may print, download, or email articles for individual use. 